precise risk of leaving it untreated is not known: following up an unselected population of patients with aneurysms until they rupture has not commended itself as a project. Size is the single factor which correlates best with risk of rupture. Rutherford's review of published work concluded that the five year risk of rupture in a $4 \mathrm{~cm}$ aneurysm (barely twice the normal diameter of the aorta) is less than $15 \%$, whereas at 8 $\mathrm{cm}$ it is greater than $75 \% .^{3} \mathrm{~A}$ diameter of $6 \mathrm{~cm}$ has often been quoted as a critical threshold in recommending surgery, but vascular surgeons are well aware that small aneurysms may rupture, and many do. ${ }^{4}$

In common with other arterial reconstruction operations aneurysm grafting is relatively new, the first aortic replacement being reported by Dubost and colleagues in $1952 .{ }^{5}$ Some surgeons operating today can remember machining their first bifurcation grafts out of nylon shirts. Modern Dacron prostheses are strong, reliable, and biologically inert; they function indefinitely and give rise to problems only in the rare event of their becoming infected. In comparison with those inserted for obliterative disease grafts for aneurysm are not prone to thrombosis. Suture materials have greatly improved too, and surgical techniques have become simpler and safer.

The operative mortality after elective resections of aneurysms in specialised units is now well below 5\%-which compares favourably, for example, with surgery of the colon-but the prognosis after rupture is grave. The patient may not survive to reach hospital-and we do not know how many deaths are certified in the community as due to myocardial infarction or other causes of collapse. If, however, he or she reaches hospital and lives through the admission procedures and the journey to the operating theatre the patient may then have a $50-75 \%$ chance of survival, provided that a team experienced in vascular surgery is available. ${ }^{6}$

Too many aneurysms are dealt with after they have ruptured rather than before. This message has recently been reinforced by Fielding and colleagues. ' In reporting an excellent survival rate of $57 \%$ in their series of patients with ruptured aneurysms treated surgically in Birmingham between 1960 and 1981 they noted that the proportion who had ruptured aneurysms $(36 \%)$ had not changed over the period despite the fact that most patients had had symptoms before rupture. The pattern of symptoms before rupture is not clear, and we do not know how many of their patients had actually reported the symptoms to their doctor.

Nevertheless, if a middle aged or elderly patient presents with an unexplained pain in the abdomen, flank, or back aneurysm must be considered as a possible cause. The nature, severity, and site of the pain depend on the direction in which the aneurysm expands and what structures it compresses or erodes. The pain may be continuous or intermittent. Arteriorly it may radiate to the iliac fossa or groin, usually the left. Posteriorly it is usually a midlumbar pain, which may radiate to the sacrum or mimic the commoner causes of root pain. Sometimes the patient notices a pulsating lump, but in an obese patient even a large aneurysm may be difficult to feel, and in a person of normal build it takes a practised and careful hand to distinguish between a small aneurysm and an aorta which is tortuous or pushed forward by a lordotic spine. Doctors sometimes forget that the aorta bifurcates at the level of the umbilicus, so the swelling may be felt well up in the epigastrium. A tender aneurysm suggests actual or imminent rupture and is an indication for urgent referral to a vascular surgeon.

Aortic aneurysm is a diagnosis made essentially by physical examination. A plain radiograph of the abdomen, especially a lateral view, will in most cases show the characteristic rim of calcification. If more information is needed ultrasound and CT scan will provide it. Aortography should be reserved for preoperative assessment, and not all vascular surgeons regard it as desirable even then. Others believe that the operation may be better planned if information is available on the state of the renal, mesenteric, iliac, and distal vessels.

The decision whether to recommend operation may be difficult for the patient who is infirm or elderly. It should be made in the light of the surgeon's awareness of his operative mortality and with the important help of an anaesthetist's opinion. If the surgeon is in doubt the patient may be followed up in the outpatient clinic. The development of symptoms or enlargement, measured by serial ultrasound scans, will invite a reappraisal.

The results of operating on a patient with a ruptured aneurysm may be very rewarding. The lucky survivor will return to a life which does not differ in quality or duration from that of his contemporaries in the general population. ${ }^{8}$ Nevertheless, the cost in terms of emergency and intensive care resources is great. By the time the patient had been admitted, assessed, transferred to theatre, resuscitated, anaesthetised, operated on, and, if surviving, safely installed in the intensive care unit some six hours or more will have passed. Many a team, as they toil through the night, knowing that there is a full day's work to follow, cannot but reflect how much better it would have been-for the patient and for themselves-if the operation had been done earlier in the calm and safety of a planned elective list.

Consultant Surgeon,
Royal Infirmary,
Edinburgh EH3 9YW

C VAughan Ruckley

1 Carlsson J, Sternby NH. Aortic aneurysms. Acta Chir Scand 1964;127:466-73.

Information Services Division of the Common Services Agency. Scottish hospital in-patient statistics. Annual reports 1972-82. Scottish Home and Health Department.

Rutherford RB, ed. Vascular surgen. Philadelphia: WB Saunders, 1984:755-71.

4 Darling RC. Ruptured arteriosclerotic abdominal aortic aneurysms. Am f Surg 1970;119:397-403. Dubost C, Allary M, Oeconomos N. Resection of an aneurysm of the abdominal aorta. Arch Surg 1952;64:405-8

6 Hicks GL, Eastland MW, DeWeese JA, et al. Survival improvement following aortic aneurysm surgery. Ann Surg 1975;181:863-9.

Fielding JWL, Black J, Ashton F, Slaney G. Ruptured aortic aneurysms: postoperative complications and their aetiology. Br f Surg 1984;71:487-91.

8 Sheranian L, Edwards GE, Kirklin GE. Late results in 110 patients treated by resectional replacement by aortic graft. Surg Gynecol Obstet 1959;109:309-14.

\section{A need for new converting enzyme inhibitors?}

Efficacy and freedom from side effects are the ultimate determinants of drug prescribing-and especially in hypertension, with most patients initially asymptomatic and yet requiring treatment for life. $\beta$ antagonists and diuretics used individually control blood pressure adequately in little more than half of all patients, and both of these groups of drugs have untoward effects, metabolic and symptomatic, in at least some people. Often a third drug is needed, but the same problems with efficacy and side effects leave no room for complacency. ${ }^{1}$

Captopril-the first orally active inhibitor of angiotensin converting enzyme-has contributed substantially to the 
management of hypertension and also of heart failure..$^{2-4}$ Its antihypertensive action when used as sole treatment appears to match that of $\beta$ antagonists, while the combination with a diuretic may greatly enhance the effect. Furthermore, captopril may avoid or even correct some of the adverse metabolic and symptomatic reactions seen with other agents. Not surprisingly, then (as was the case earlier with $\beta$ antagonists and thiazides), several newer converting enzyme inhibitors are under development. Can any advantages be expected with these more recent drugs?

Captopril is a potent inhibitor of converting enzyme, and there seems little scope for improvement here. Its duration of action on the renin-angiotensin system in peripheral blood is short; even so, twice daily administration usually controls the blood pressure over 24 hours. Nevertheless, an agent with a longer duration of action allowing once daily dosing would have an advantage - at least in convenience to the patient.

Complete absence of side effects must perhaps remain an elusive ideal. Though captopril is usually tolerated remarkably well, it does possess side effects, some of which are serious. The frequency and severity of these adverse reactions were exaggerated by the use in early days of excessively high doses-and this hindered the acceptance of the drug into clinical practice. Captopril contains a sulphydryl group and depends on the kidney for elimination. How many of the untoward effects-which include rashes, disturbances of taste, fever, arthralgia, leucopenia, persistent cough, and proteinuria-are due to the presence of this group is not yet clear; but reduction of dosage, particularly with renal impairment, may help avoid the more serious and perhaps even some of the milder reactions. In particular, proteinuria does not seem to be related to captopril administration when low but effective doses are given to patients with normal renal function. Rashes and disturbances of taste, however-the most frequent problems with captopril-have led to withdrawal of treatment from respectively $1.4 \%$ and $0.5 \%$ of patients with normal renal function even when receiving the lower doses now recommended. ${ }^{5}$ Hence a converting enzyme inhibitor not dependent mainly on the kidney for elimination and perhaps without the sulphydryl group might have advantages.

Newer agents require to be exposed to the same widespread prescribing as captopril before any theoretical advantages they may have can be confirmed. Of the newer agents, only enalapril has been used sufficiently extensively for even tentative comparison. ${ }^{56}$ Enalapril maleate (MK 421 ) is the readily absorbed ethyl ester of the active drug enalaprilat (MK 422). ${ }^{7}$ Given once daily enalapril produces effective 24 hour inhibition of converting enzyme. ${ }^{8} \mathrm{~A}$ single daily dose seems adequate in treating hypertension in lowering both the plasma concentrations of angiotensin II and the blood pressure. ${ }^{7}$ As was the case with captopril the hypotensive effect of enalapril given alone appears at least as great as that of $\beta$ antagonists or diuretics and is enhanced by combination with a diuretic. ${ }^{7910} \mathrm{~A}$ particularly encouraging feature is that in one trial enalapril was found to be especially effective in reducing the systolic pressure. ${ }^{10}$ Enalapril is well tolerated by patients, a feature which is increasingly apparent with this group of drugs.

Over 2000 patients have now received enalapril. ${ }^{5}$ Loss of taste attributable to the drug has not occurred, and only $0.3 \%$ of patients have discontinued treatment because of a rash; neutropenia $\left(<300 \times 10^{6}\right.$ cells/l) has not been found. ${ }^{5}$ Enalapril has not been shown to cause proteinuria $(>1 \mathrm{~g}$ protein per day), nor has any case of the nephrotic syndrome been reported. These observations are encouraging, ${ }^{6}$ though no direct comparison of the frequency and severity of side effects of captopril and enalapril has been published.

As would be expected from their mode of action, converting enzyme inhibitors are particularly effective in reducing the blood pressure in patients with renal artery stenosis. $^{237}$ The kidney distal to a renal artery stenosis appears, however, to be heavily dependent on an intrarenal action of angiotensin II to maintain the glomerular filtration, excretion of urea, and distal renal artery pressure. These actions are lost with converting enzyme inhibition. Thus, though effective in controlling renovascular hypertension and in many instances improving overall renal function, this form of treatment usually adversely influences the afflicted kidney. In the rare circumstances when renal artery stenosis is bilateral or affects a sole remaining kidney renal failure may result, especially when diuretics are given concurrently. " Fortunately the renal failure is reversible if treatment is withdrawn. This problem is likely to be encountered with all converting enzyme inhibitors.

Some criticism may be levelled at the drug industry for apparent dissipation of effort in developing numerous agents with essentially the same mode of action, so well illustrated earlier by the $\beta$ antagonists. Nevertheless, the availability of a range of drugs with differing pharmacokinetics may be no bad thing. Cardioselective $\beta$ blockers, such as atenolol and metoprolol, offer distinct therapeutic advantages. ${ }^{12}$ But detection of idiosyncratic and potentially disastrous consequences of any drug (as was so well illustrated by the sad story of practolol) will come only from continued vigilance when the agent is widely prescribed. ${ }^{13}$ In such circumstances the availability of alternative drugs with a similar action is crucial. As a group, converting enzyme inhibitors are likely to be effective and well tolerated; the physician is soon to be faced with a wide choice.

S G BALL

British Heart Foundation senior research fellow

J I S ROBERTSON Consultant physician

MRC Blood Pressure Unit,

Western Infirmary,

Glasgow G11 6NT

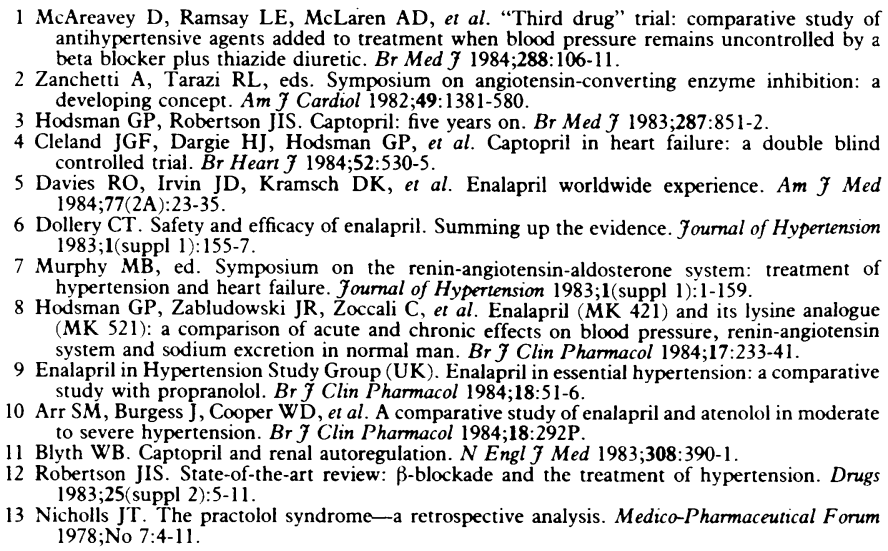

McAreavey D, Ramsay LE, McLaren AD, et al. "Third drug" trial: comparative study of beta blocker plus thiazide diuretic. Br Med f 1984;288:106-11. anchetti A, Tarazi RL, eds. Symposium on angiotensin-convert

Capl 1982;49:1381-580.

4 Cleland JGF, Dargie HJ, Hodsman GP, et al Captopril in $1983,287.851-2$ double blind controlled trial. Br Heart f 1984;52:530-5. Datros

irphy MB, ed. Symposium on the renin-angiotensin-aldosterone system: treatment of and heart failure. Foumal of Hypertension


study with propranolol. Br $\mathcal{F}$ Clin Pharmacol 1984;18:51-6.

Arr SM, Burgess J, Cooper WD, et al. A comparative study of enalapril and atenolol in moderate Blyth WB. Captopril and renal autoregulation. N Engl Y Med 1983;308:390-1.

obertson JIS. State-of-the-art review: $\beta$-blockade and the treatment of hypertension. Drugs 1978;No $7: 4-11$. 